\title{
Parallel and distributed neural models of the ideomotor principle: An investigation of imitative cortical pathways
}

\author{
Eric L. Sauser*, Aude G. Billard \\ LASA Laboratory, Ecole Polytechnique Fédérale de Lausanne, EPFL, Station 9, 1015 Lausanne, Switzerland
}

\begin{abstract}
Humans' capacity to imitate has been extensively investigated through a wide-range of behavioral and developmental studies. Yet, despite the huge amount of phenomenological evidence gathered, we are still unable to relate this behavioral data to any specific neural substrate. In this paper, we investigate how principles from psychology can be the result of neural computations and therefore attempt to bridge the gap between monkey neurophysiology and human behavioral data, and hence between these two complementary disciplines.

Specifically, we address the principle of ideomotor compatibility, by which 'observing the movements of others influences the quality of one's own performance' and develop two neural models which account for a set of related behavioral studies [Brass, M., Bekkering, H., Wohlschläger, A., \& Prinz, W. (2000). Compatibility between observed and executed finger movements: comparing symbolic, spatial and imitative cues. Brain and Cognition 44, 124-143]. We show that the ideomotor effect could be the result of two distinct cognitive pathways, which can be modeled by means of biologically plausible neural architectures. Furthermore, we propose a novel behavioral experiment to confirm or refute either of the two model pathways.
\end{abstract}

(C) 2006 Elsevier Ltd. All rights reserved.

Keywords: Neural mechanisms of imitation; Ideomotor principle; Neural field computation

\section{Introduction}

Human capacity to imitate has been extensively investigated through a wide range of behavioral and developmental studies (see Billard, 2002 for a review). Yet, despite the huge amount of phenomenological evidence gathered, we are still unable to relate this behavioral data to any specific neural substrate. Particularly informative in the attempt to resolve this issue was the neurological evidence for the existence of a common neural substrate devoted to the recognition and production of movements, the so called mirror neuron system (see Decety \& Sommerville, 2003; Iacoboni et al., 1999; Rizzolatti et al., 2001 for recent reports on this system in monkeys and humans). While the mirror neuron system offers an exciting line of study, it has yet to be shown how this circuit, in connection with other well-known neural circuits for visual representation of motion and for motor control, may explain the behavioral data on imitation.

\footnotetext{
* Corresponding author.

E-mail address: eric.sauser@epfl.ch (E. Sauser).

$U R L:$ http://lasa.epfl.ch.
}

0893-6080/\$ - see front matter (C) 2006 Elsevier Ltd. All rights reserved. doi:10.1016/j.neunet.2006.02.003
Several computational studies using different approaches have already attempted to address the issue of the mirror neuron system. A comprehensive review of these studies can be found in Oztop, Kawato, and Arbib (2006). One of the most influential approaches is based on motor control theory (Billard \& Mataric, 2001; Demiris \& Hayes, 2002; Oztop et al.; Wolpert, Doya, \& Kawato, 2003), which considers the tight link between motor execution and action observation. In this approach, a set of predictive inverse and forward models allows an observed movement to be compared with entries in the observer's motor repertoire. When a sufficiently similar action is found, its execution is facilitated. Our work is more in line with that of Arbib, Billard, Iacoboni and Oztop (2000), which attempts to uncover the neural pathways at the origin of human imitation capabilities. Our approach, however, is strongly multidisciplinary, in that its main sources of inspiration come from both psychological theories and neuroscience. We investigate how the former's principles can be the result of neural computations and therefore attempt to bridge the gap between monkey neurophysiology and human behavioral data, and hence between these two complementary disciplines.

In this paper, we address the principle of ideomotor compatibility, by which 'observing the movements of others influences the quality of one's own performance' (Brass, 
Bekkering, \& Prinz, 2001; Brass, Bekkering, Wohlschäger, 2000; Heyes, Bird, Johnson, \& Haggard, 2005; Kilner, Paulignan, \& Blakemore, 2003), and develop two neural models which account for a set of related behavioral studies (Brass et al., 2000). We show that the ideomotor effect could be the result of two distinct cognitive pathways, which can be modeled by means of biologically plausible neural architectures. Furthermore, we propose a novel behavioral experiment to confirm or refute either of the two model pathways. In Section 2, we briefly recall the experiment by Brass et al.

\subsection{Brass et al. experiment}

In their experiment Brass et al. (2000), used a stimulusresponse (SR) paradigm to verify two hypotheses of the ideomotor theory. These two hypotheses are based on the neural correlate that the human brain appears to possess highly specialized neural circuits devoted to the recognition of movements performed by others and that these circuits are likely to be shared by the motor preparation circuits (Decety \& Sommerville, 2003; Iacoboni et al., 1999). The first of the hypotheses states that if a subject was requested to respond to the motion of a demonstrator then he would experience a motor facilitation, giving faster reaction times compared to if the subject was asked to make the same movement in response to a spatial cue. The second hypotheses states that the facilitatory effect would be greater if the movements of the demonstrator and subject were very similar (ideomotor compatible) than if they were of a different type (ideomotor incompatible).

The experimental setup comprised of three independent binary variables, leading to eight conditions plus four baseline conditions. The experimental stimuli consisted of a combination of a finger-lifting movement (either index or middle finger) and of a spatial cue consisting of a cross painted on the corresponding or opposite fingernail (see Fig. 1). The subjects reaction times (RTs) were measured while they were asked to

(a)
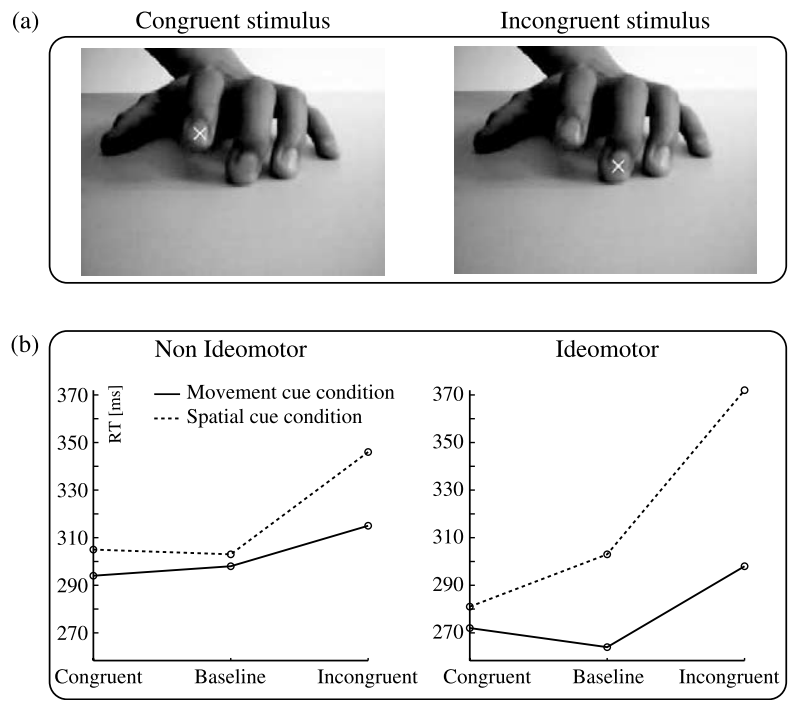

Fig. 1. (a) Examples of congruent and incongruent stimuli used by (Brass et al., 2000) in their experiment; (b) reaction times observed in the original experiment. respond to the various stimuli by moving the finger that was the closest to either cue (e.g. by moving their index finger for a demonstration of the index finger or for the presentation of a cross on the demonstrator's index fingernail).

These instructions determined the first experimental variable, the relevant stimulus dimension. Furthermore, an interfere paradigm was used in order to examine the effect of the presentation of congruent or incongruent ${ }^{1}$ stimuli against a baseline condition in which only the relevant stimulus was presented to the subject. Finally, the experiment was varied in order to examine the effect of ideomotor similarity between observed and executed movements. In one case, the subjects were asked to lift their finger (ideomotor compatible) and in the second they were asked to produce a finger-tapping movement (ideomotor incompatible).

The results, shown ${ }^{2}$ in Fig. 1, were in agreement with the hypotheses. Indeed, responses to finger movements were faster than responses to spatial cues, and ideomotor compatible pairs of observed/executed movements generally produced better RTs. Moreover, typical facilitatory and interference effects were observed between congruent and incongruent conditions, respectively. Next, we present two neural models, which account for these results.

\section{Models}

Our modeling approach starts with the well-accepted hypothesis that the brain uses parallel pathways to process multimodal information. This so called parallel distributed processing (PDP) framework has been successfully applied in explaining a variety of effects observed during stimulusstimulus and stimulus-response compatibility experiments (Erlhagen \& Schöner, 2002; Hasbroucq \& Guiard, 1991; Kornblum, 1994; Zhang et al., 1999). In these models, the information passes through a layered network organization, usually consisting of the perceptual, decisional and motor preparatory stages of computation. Generally, multimodal perceptual information is processed separately and simultaneously in a first stage and is then combined within the other layers depending on the nature of the information. This fusion of information within a common layer has sometimes been referred to as the dimensional overlap (Kornblum, 1994), which gives a measure of the degree to which sets of items are perceptually, structurally or conceptually similar. This principle allows perceptually similar information to be merged into a common neural substrate and such a mechanism has proved useful in explaining a wide range of human behaviors (Erlhagen \& Schöner, 2002; Hasbroucq \& Guiard, 1991; Kornblum, 1994; Zhang, Zhang, \& Kornblum, 1999). In this

\footnotetext{
${ }^{1}$ Congruent condition: a left (right) finger movement with a cross on the left (right) fingernail. Incongruent condition: a left (right) finger movement with a cross on the right (left) fingernail.

${ }^{2}$ As the ideomotor variable was tested among two distinct groups of subjects, we shifted the reaction times to make the baseline condition in the spatial cue task coincide in both experiments, since this is the only case in which both experimental conditions are identical.
} 
paper, we develop a similar type of neural architecture to account for the visuo-motor flow at the basis of our simple imitation task. We note that the classical PDP layered architecture was adapted in order to account for the evidence that the perception of movements of others and the motor representation of one's own motions share a common neural substrate (Decety \& Sommerville, 2003; Iacoboni et al., 1999; Rizzolatti, Fogassi, \& Gallese, 2001).

The two models which we propose here, mainly rely on the same principles and so we will describe them first, then we will explore the fundamental differences in the network connectivity between these two models, and finally we will present their neural implementation.

\subsection{Models principles}

The two proposed networks are illustrated in Fig. 2. They can be split into three major parts: the perceptual, decisional and motor preparatory layers, which respective tasks are to represent visual information, to determine the right response to
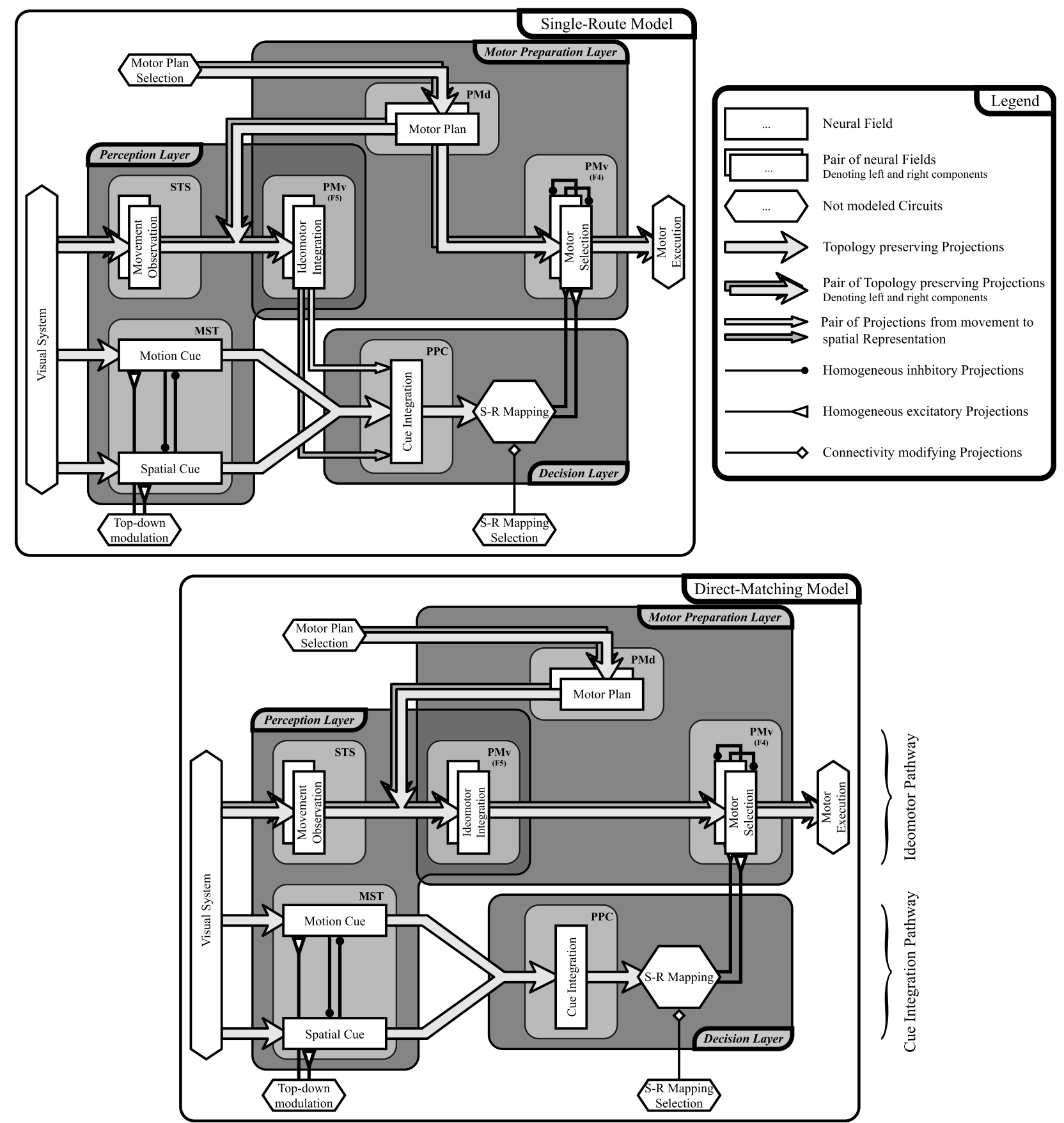

Fig. 2. Schematic of the two architectures proposed to model the behavioral effect related to the ideomotor principle. At the top, the single-route architecture assumes that all the processed stimuli interact within the same integration layer. At the bottom, two separate pathways are involved in the computation. The cue integration pathway accounts for the selection across spatial cues, whereas the ideomotor pathway influences the motor selection mechanism by means of a direct connectivity. 
external stimuli, and to prepare and trigger motor execution. Moreover, the processing of the task instructions will not be explicitly explained here, as we assume it to be localized in frontal cortices and to drive the network models by modulation of neural activity.

\subsubsection{Perception}

In our models, perception is only considered in its final stage, in that we assume visual information to have already been processed by highly specialized circuits and represented in a manner relevant for the task. We consider three types of visual inputs in our model. First, the motion and spatial cues encode, respectively, the location, in retinal coordinates, of the observed moving finger and of the cross-drawn on the fingernail. As this cues are encoded into spatial coordinates, we assume that these representations are located in the visual 'where stream', in areas such as the medial superior temporal cortex (MST) (Andersen, Snyder, Bradley, \& Xing, 1997) or the lateral intraparietal area (LIP) (Schadlen \& Newsome, 2001). ${ }^{3}$

Concerning the internal properties which this model region should possess, we first have to determine in what manner task instructions might act upon the perception of these two types of stimuli. Indeed, given the instructed task-relevant stimulus, the decisional process should be able to activate the correct stimulus-response association. It has been suggested that such a process is the result of a top-down modulation of cortical activity (Itti \& Koch, 2001). This principle is directly applied to the perceptual representations of our models.

Second, it has been shown in human subjects that the presentation of these two types of stimuli do not produce similar results in terms of RTs, as motion cues generally receive more attention than spatial cues (Franconeri \& Simons, 2005). Therefore, in order to account for the results of Brass et al., which also showed significantly faster reaction times for motion cues than for spatial cues, the influence of the motionrelated stimuli was given greater importance by modeling it with a greater amplitude.

Moreover, behavioral studies investigating visual perception have shown that in the presence of $N$ stimuli, the RT increases proportionally with the binary logarithm of $N$, an effect usually referred to as Hick's law (Hick, 1952). In simpler terms, the more information that is perceived, the longer the RT. In Brass et al. experiment, the baseline condition involved only one stimulus, while the normal task conditions involved two. The respective influence of both types of stimuli would, in the latter case, compete and hence slow down the overall integration process. As shown in Section 2.4.3, this effect was modeled using competitive interactions across the perceptual representations.

Finally, the third type of visual input concerns movement observation. It has been shown, in the superior temporal sulcus (STS) of the monkey, that the activity patterns of neural

\footnotetext{
${ }^{3}$ As the neural recordings were mainly obtained in monkeys we assume, throughout this paper, the homologies across brain areas between humans and monkeys (Arbib and Bota, 2003).
}

populations correlate with the observation of specific limb movements (Jellema, Maassen, \& Perrett, 2004; Perrett, Harries, Mistlin, \& Chitty, 1989). Furthermore, as movement observation has also been shown to activate the human homologue region (Decety \& Sommerville, 2003; Iacoboni et al., 1999), we assume here, the existence of neural populations in which movements performed by others, such as finger movements, are encoded. Consistently with the part of the model dedicated to motor preparation, which will be described later, and following the observation that mirror neurons fire similarly when observing or executing an action (Rizzolatti et al., 2001), we also hypothesize that this representation lies within the same frame of reference (FR) as self organized movements, i.e. within motor coordinates. As will be further explained, this property allows the model to compare these two forms of information, and hence to produce the reported effects of ideomotor compatibility.

\subsubsection{Decision and response selection}

The main task of the proposed models is to perform a selection among different sources of stimuli. As previously mentioned the information related to motion and spatial cues is supposed to follow the dorsal 'where stream'. Therefore, this perceptual information is then fed to a decisional process, whose neural substrate seems to be located in the posterior parietal cortex (PPC), a region that was shown to be the locus of visual target selection (Andersen et al., 1997; Schadlen \& Newsome, 2001).

Furthermore, as soon as the decision process is performed, the brain must transform information from stimulus space to motor space by means of stimulus-response associations (SRA) (Wilimzig \& Schöner, 2005). It is not within the scope of this paper to explain how such mechanism can be modeled but a plausible answer has been proposed in Wilimzig and Schöner (2005). The proposed solution consists of a complete representation of all the combinations, which can be mapped using the stimulus and response space. When a stimulus is presented, it activates all the combinations it corresponds to and the response is then chosen according to the favorite SRA, or according to another mapping that can be favored by the task instructions. In our present models, we have assumed that such a transformation is carried out by hard-wired connections from the decisional process to the final motor preparatory stage. In addition, this connectivity can be modified by the task instructions mediated by the prefrontal cortex, so that different SRA's can be produced. However, associations that are different from the mirror responses, i.e. a left/right movement in response to a left/right stimulus, would result in longer reaction times. Indeed, behavioral studies addressing this issue have shown a constant increase of the RT under such conditions, and have thus suggested that processing in higher cognitive areas is involved (Hasbroucq \& Guiard, 1991; Hedge \& Marsh, 1975; Proctor \& Pick, 2003).

\subsubsection{Motor preparation}

The motor preparation layer that we consider consists of three areas, coding, respectively, for the motor plan of each 
finger, for the final motor selection, and for the shared representation between movement observation and motor execution that we will designate as the ideomotor integration area. The motor plans are fed either directly or indirectly through the ideomotor area to the final motor selection layer which is waiting for the execution signal coming from the decisional layer (see Fig. 2).

By definition, the ideomotor region is where information related to both movement observation and movement preparation overlap and hence is the area responsible for ideomotor effects. Indeed, the ideomotor theory predicts that the ease with which a stimulus can be transformed into an action depends on the similarity between the observed event and the executed action (Brass et al., 2000; 2001; Greenwald, 1970; Wohlschläger et al., 2003). As suggested by Greenwald, (1970), the ideomotor theory relies on the assumption that sensory feedback is compared with the response image.

We then follow the neurological evidence of the direction along which both intended and observed movement are represented within populations of neurons (Cisek \& Kalaska, 2005; Perrett et al., 1989; Schwartz et al., 1988) and we associate to each finger a population coding for the imitator's intended motor plan and for the observation of the corresponding demonstrator's finger movement. These populations are, respectively, assumed to be located in the dorsal premotor cortex (PMd) (Rizzolatti et al., 2001) and STS (Jellema et al., 2004; Perrett et al., 1989). Both populations then project onto a single motor preparatory population in the area F5 of the ventral premotor cortex (PMv), a region where mirror neurons were found (Rizzolatti et al., 2001). This latter area will evaluate the ideomotor compatibility of observed and intended movements. High ideomotor compatibility happens when the demonstrator and the imitator move the same finger in the same direction. Conversely, low ideomotor compatibility is reached when demonstrator and imitator produce movements of different fingers in the opposite direction.

\subsection{Models architecture}

\subsubsection{Single-route model}

Following a PDP-like architecture as usually proposed in experimental psychology (Hasbroucq \& Guiard, 1991; Kornblum, 1994; Zhang et al., 1999), we first designed a model, which consisted of a typical layered model where the spatial cue, the motion cue, and the ideomotor compatibility cue interact together within the decisional layer. The location of the winning stimuli is then used to trigger movement execution ${ }^{4}$ in the final motor selection area (located in area F4 of $\mathrm{PMv}$ region known to encode motor programs before

\footnotetext{
${ }^{4}$ The motor execution part will not be modeled in this paper, as the execution time is assumed to be constant under all conditions.
}

execution (Cisek \& Kalaska, 2005)) by means of stimulusresponse mapping.

\subsubsection{Direct-matching model}

Second, we propose an alternative model illustrated at the bottom of Fig. 2. As suggested by the direct-matching hypothesis (Meltzoff \& Moore, 1997; Rizzolatti et al., 2001), stating that the brain structures involved directly in action observation interact with those concerned with the corresponding motor execution, a second architecture was built, in which two distinct information pathways and two selection processes coexist. The first pathway is strictly concerned with integrating spatial visual information such as spatial cues and spatial localization of motion. This corresponds to the dorsal 'where' stream, that is assumed to originate from the MST region (Andersen et al., 1997). Separately, the so-called ideomotor pathway integrates the representation of the motor plans together with the representation of movements performed by others. This pathway is assumed to follow the ventral 'what' stream, which passes through STS and then projects onto the so-called mirror areas in PMv (Rizzolatti et al., 2001). Both these pathways finally converge toward the final motor selection area, which has to execute the correct response instructed by the decisional layer, under the influence of the ideomotor pathway.

\subsection{Dynamic neural field model}

The neural implementation of the two models is inspired by neurophysiological evidence suggesting a continuous representation of stimuli in feature maps (Ashbridge et al., 2000; Cisek \& Kalaska, 2005; Schwartz et al., 1988). In such neural populations, neurons generally respond to external stimuli with broad tuning curves of activity. Therefore, we adopted the dynamic neural field approach (Amari, 1977; Erlhagen \& Schöner, 2002; Zhang, 1996) which integrates the principles of continuous representations endowed with a metric (Erlhagen \& Schöner, 2002; Wilimzig \& Schöner, 2005), and can account for temporal dynamics of stimuli interactions. We then assume that each variable considered in the models is represented within a distinct neural population.

Before we start, we should stress that while we aim to explain the difference in reaction times between two behavioral processes, it is beyond the scope of the present paper to account for the precise time course of the neural and sensorimotor processes. Thus, in the remainder of this paper we will report on qualitative effects, measured as the relative time required for a given network to perform the task. For convenience, this data was fitted to the original reaction times in order to be able to compare simulation results and behavioral data on the same time scale (see Appendix A).

\subsubsection{Definition}

Formally, a neural field is composed of a continuous set of neurons firing maximally for a specific value $\theta$ in the parameter space. This unimodal type of activation is illustrated in Fig. 3a. In order to avoid the problem of boundary effects, preferred 

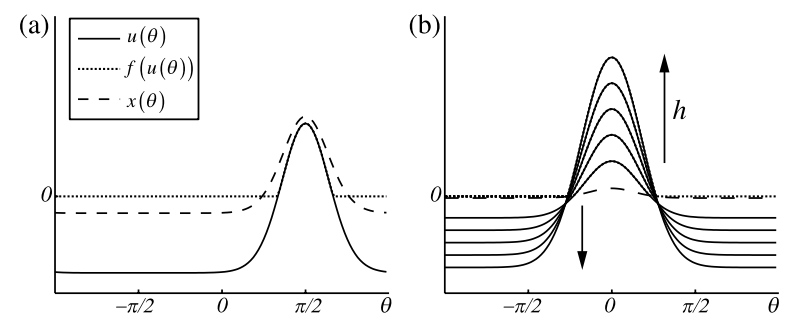

Fig. 3. (a) Illustration of a neural field subjected to a spatial input localized at $\pi / 2$. (b) The population profile of activity is gain-modulated by means of an external homogeneous input $h$. This effect is shown for various values of $h$.

values of $\theta$ are uniformly distributed along a circular space such that $\theta \in[-\pi, \pi]$. It should be emphasized here that any kind of information could be encoded by means of a neural field. Indeed, a unidimensional variable $y$ defined in an arbitrary domain can be transformed into the considered space using a mapping function $f(y) \rightarrow \theta$. In the present case, spatial and motion cues are assumed to be encoded in retinal coordinates, whereas movement observation and motor plans are represented in motor coordinates using the movement direction as a basis for each finger (Cisek \& Kalaska, 2005; Schwartz, Kettner, \& Georgopoulos, 1988).

The network is fully connected by means of recurrent synaptic weights $W_{i}^{R}$, exhibiting symmetry, rotation invariance and center-surround excitation-inhibition characteristics. The network's dynamic follows

$\tau \dot{u}_{i}(\theta, t)=-u_{i}(\theta, t)+x_{i}(\theta, t)+h_{i}(t)+\oint W_{i}^{R}(\theta-\phi) f\left(u_{i}(\phi, t)\right) \mathrm{d} \phi$

where $u_{i}(\theta, t)$ is the membrane potential of the neuron with preferred value $\theta$ at time $t$, belonging to the population $i$. The non-linear activation function is defined as $f(y)=\max (0, y)$. $x_{i}(\theta, t)$ corresponds to the external input and $h_{i}(t)$ to a global homogeneous input. The weight linking two neurons, with preferred directions $\theta$ and $\phi$, is given by a periodical Gaussian profile defined as

$W_{i}^{R}(\theta-\phi)=\frac{\alpha_{i}}{\kappa}\left[\exp \left(\frac{\cos (\theta-\phi)-1}{2 \sigma^{2}}\right)-1\right]$

where $\alpha_{i}$ and $\sigma$ are, respectively, the amplitude and variance of the weights. $\kappa=1-\mathrm{e}^{-\left(1 / \sigma^{2}\right)}$ ensures that the weights are inhibitory and bounded, i.e. $W_{i}^{R}(\theta-\phi) \in\left[-\alpha_{i}, 0\right]$. As shown in Section 2.4.2, these recurrent connections define the metric and the interaction strength between spatially localized inputs such that close stimuli cooperate in the representation, whereas far ones compete and interfere.

\subsubsection{External inputs}

Each network $i$ can receive an external input $x_{i}(\theta, t)$ consisting of a periodic Gaussian $g_{i}$ localized at $\phi_{i}$ in the neural space, such that

$g_{i}(\theta, t)=\frac{\beta_{i}(t)}{\kappa}\left[\exp \left(\frac{\cos \left(\theta-\varphi_{i}\right)-1}{2 \sigma^{2}}\right)-\eta\right]$
$x_{i}(\theta, t)=g_{i}(\theta, t)$ where $\beta_{i}(t)$ is the input amplitude, and $\eta$ is a normalization factor, ensuring that $\int g_{i}(\theta, t) \mathrm{d} \theta=0$. As illustrated in Fig. 3a, an external input produces a unimodal increase of the population potential. In the experiments reported in Section 3, the external cues (e.g. the cross and the finger movement) are being modeled as external inputs located at positions $\varphi_{i}$ driving the dynamics of the their corresponding populations $i$.

\subsubsection{Projection between populations}

In addition to the external inputs mentioned in Section 2.3.2, a neural field can be subjected to synaptic projections arising from other populations. The projections between two neural fields can be of two types. First, topology preserving projections ensure that a localized peak of activity in the source population also produces a localized input in the target population. Secondly, homogeneous projections uniformly modulate the target population proportionally to the global activity of the source field.

Topology preserving projections are made through synaptic weights $W_{i \rightarrow j}^{P}(\theta-\phi)$ between neuron with preferred directions $\theta$ and $\phi$ of the source and target population, $i$ and $j$, respectively. They are defined by

$W_{i \rightarrow j}^{P}(\theta-\phi)=\frac{\gamma_{i \rightarrow j}}{\kappa}\left[\exp \left(\frac{\cos (\theta-\phi)-1}{2 \sigma^{2}}\right)-\eta\right]$

such that the external input $x_{j}(\phi, t)$ of the target population becomes

$x_{j}(\phi, t)=\oint W_{i \rightarrow j}^{P}(\theta-\phi) f\left(u_{i}(\theta, t)\right) \mathrm{d} \theta$

where $\gamma_{i \rightarrow j}>0$ is the strength of the projection weights.

Homogeneous projections consist of a uniform modulation that could either be excitatory or inhibitory. They are defined such that

$h_{j}(t)=W_{i \rightarrow j}^{M} \oint f\left(u_{i}(\theta, t)\right) \mathrm{d} \theta$

where $W_{i \rightarrow j}^{M}$ corresponds to the weight of the homogeneous modulation from initial population $i$ to the target $j$.

\subsubsection{Stimulus-response and inverse mapping}

The stimulus-response projections from the cue integration layer uniformly modulate either of the sub-areas of the motor selection layer and are defined by

$h_{j}(t)=\oint W_{i \rightarrow j}^{P}\left(\theta-\phi_{j}\right) f\left(u_{i}(\theta, t-\Delta)\right) \mathrm{d} \theta$

where $i$ applies only for the population cue integration and $j$ for motor selection left and right. $\Delta$ corresponds to an artificial processing time taken by that operation which is zero when the mapping is natural (see Section 2.1.2), and otherwise is set according to behavioral literature (Proctor \& Pick, 2003) (see Appendix A).

As can be seen in Fig. 2, the Single-Route model possesses connections from the ideomotor area to the cue integration area. As the representation of both systems differs, one has to 
define an inverse transformation of that defined previously. It is given by adding localized inputs $g_{i}(\theta, t)$ to the cue integration area similarly to Eq. (3)

$x_{j}(\theta, t)=\sum_{i} g_{i}(\theta, t)$

where $i \in\{$ ideomotor integration left, right $\}$ and $j=c u e$ integration. The amplitude $\beta_{i}(t)$ of the input $g_{i}(\theta, t)$ as defined in Eq. (3) is equal to

$\beta_{i}=\gamma_{i \rightarrow j} \oint f\left(u_{i}(\theta, t)\right) \mathrm{d} \theta$

and its location $\varphi_{i}$ corresponds to that of the motion cue.

\subsection{Network properties}

In the following paragraphs, we will summarize briefly the properties of neural fields relevant to our models. Since the main task of the proposed models is to perform a selection among different stimulus sources we start by showing how such a selection mechanism can be produced within a single neural field. We will then illustrate how a similar mechanism can be applied to model the Hick's law effect on spatial and motion cue integration during the early sensory processing stage.

\subsubsection{Stimulus enhancement}

In order to choose one of two stimuli, we will use a metric which gives a measure of the relevancy or importance of each stimulus (Erlhagen \& Schöner, 2002). Here, we follow (Pouget et al., 2003) and take the amplitude of the stimulus as a measure of its relevancy. Therefore, in the task we consider here, when two stimuli convey an equal amount of information the relevant (task-related) stimulus must first be enhanced in order for it to be selected. Such an increase in the stimulus amplitude can be induced by simply increasing the homogeneous excitatory input $h$ in Eq. (3). $h$ acts as an attentional gain, by modulating the shape of the network's activity profile (Salinas \& Abbott, 1996; Sauser \& Billard, 2005) (see Fig. 3).
In the experiments reported in Section 3, the models will be driven by the task instructions that will help select the relevant stimulus, by enhancing the corresponding brain area by means of cortico-cortical connections, represented in our model as a top-down modulatory input $h^{\mathrm{T}}$ such that

$h_{i}(t)=\delta(i, j) h^{\mathrm{T}} \quad$ and $\quad \delta(i, j)= \begin{cases}1 & i=j \\ 0 & i \neq j\end{cases}$

where $i \in\{$ spatial cue, motion cue $\}$ and $j$ is the index of the neural field corresponding to the task-relevant stimulus dimension.

\subsubsection{Selection, cooperation and interference}

In order to select from two competing stimuli within a single neural population, one must apply sufficiently strong recurrent weights across neurons within the neural field (Erlhagen \& Schöner, 2002; Kopecz \& Schöner, 1995). This selection process is illustrated in Fig. 4. Two competing stimuli of equivalent strength are simultaneously presented to the network (initially at rest) at different locations. The network converges toward an unstable state composed of two distinct regions of activity. Because of the interaction across these two regions, even a small variation in intensity of one of the two inputs is sufficient to break the symmetry and results in a single peak of activity.

Both the cue and ideomotor integration fields work on this principle. As two inputs interact within the same neural population, if they are close enough, in the neural population, they will cooperate, otherwise they will interfere.

\subsubsection{Competition between populations}

As mentioned in Section 2.1.1, the results in Brass et al. (2000) suggest an occurrence of the Hick's law effect in their experimental paradigm. This suggests a neural process that cannot solely be the result of the selection mechanism as previously described. Indeed, the aforementioned selection process produces a clear facilitatory effect for the congruent conditions, an effect already demonstrated by Erlhagen and

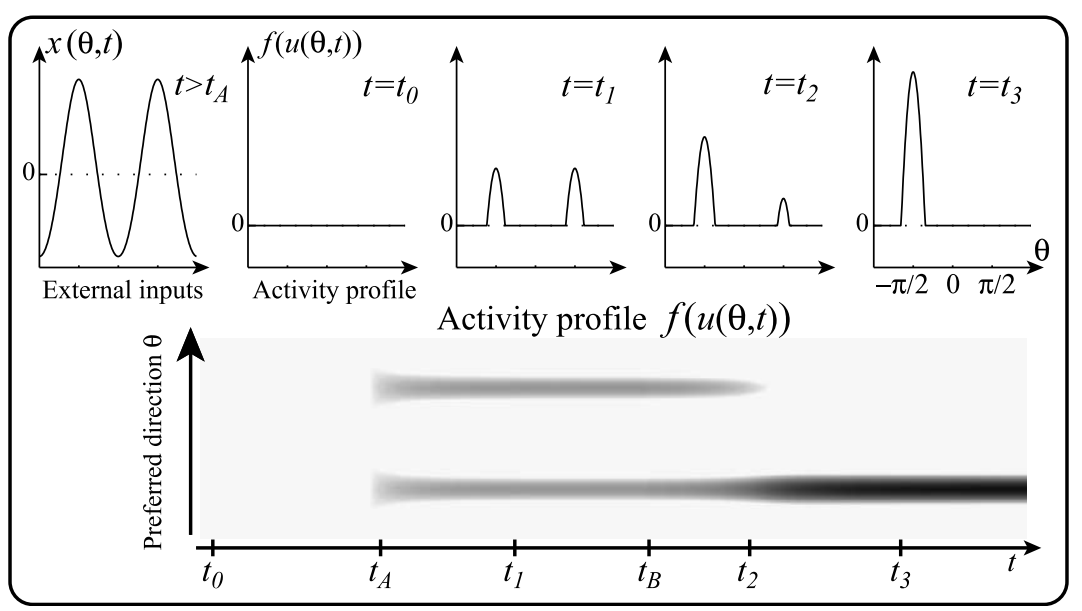

Fig. 4. Time $t_{A}$ : two equal and competing stimuli are presented to the network. The network converges toward an unstable state where both stimuli are represented. Time $t_{B}$ : a short perturbation is induced in the first stimulus. This is enough to break the symmetry and leads to a single blob of neural activity. 

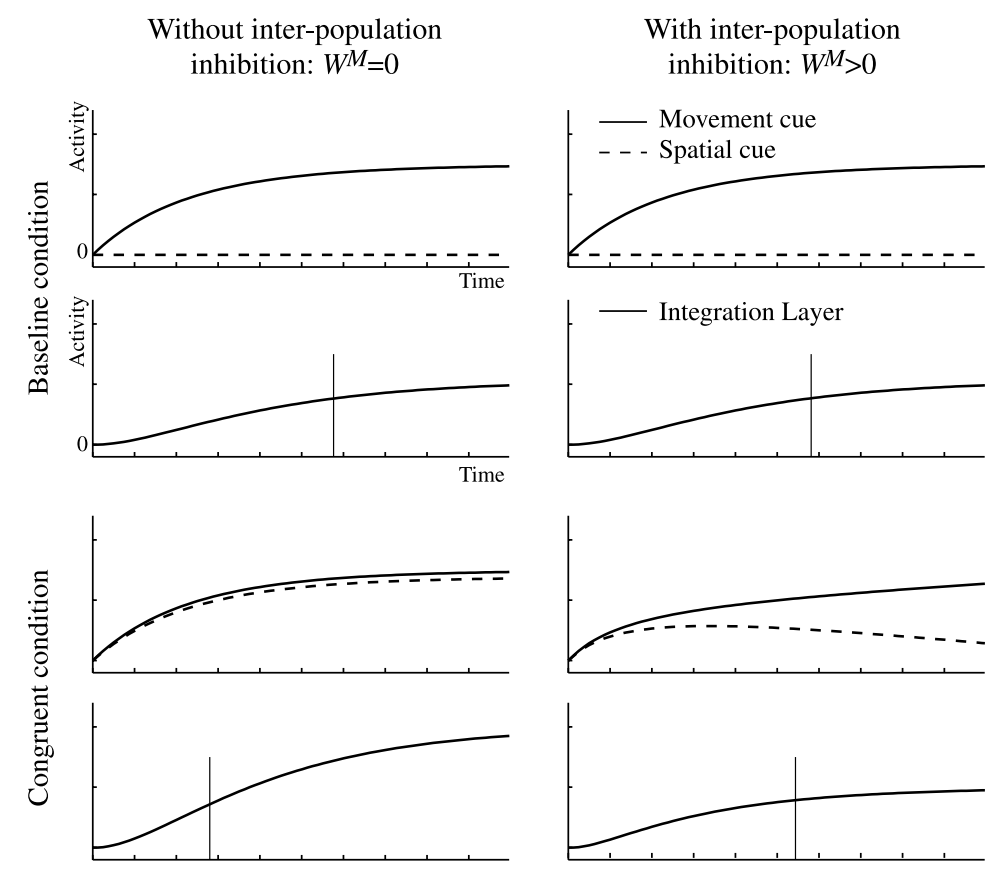

Fig. 5. Illustration of the mechanism reducing the RT difference between the congruent and baseline conditions. For each neural population involved here, only the neuron with maximal activation is shown. In each subplot, the top panel shows the evolution of activity of the perceptual cues' representations while the bottom panel shows the cue integration layer. The vertical bar indicates the time when the cue integration layer reached a given threshold.

Schöner (2002). They also showed how Hick's law could be reproduced from interactions within the same neural layer. Here, we apply the same technique but between different stimulus representations using reciprocal inhibitory connections $W_{\text {Perc }}^{M}$ between the two neural fields corresponding to the spatial and motion cues. When two inputs are present they inhibit each other by decreasing their respective amplitudes. When there is only a single input, however, it is freely integrated without any competition. As a result, in the congruent condition, the selection layer will converge slower and the RT difference will then be decreased. This effect is illustrated in Fig. 5.

Finally, such a mechanism is, on its own, also a selection process between the representation of two distinct populations. Therefore, the mechanism described was also applied to the motor selection area containing preshapes of both the fingers' motors plans in two distinct sub-populations. The selected motor plan is then executed as soon as the global activity of its corresponding population reaches the threshold $\delta$ as defined by

$\oint f\left(u_{i}(\theta, t)\right) \mathrm{d} \theta>\delta \Rightarrow$ Motor plan $i$ is executed

where $i \in\{$ motor selection left, right $\}$.

\section{Results}

The behavioral experiments were simulated by the two models using the same initial conditions as those used by Brass et al. in their behavioral experiments (Brass et al., 2000). The results are shown in Fig. 6, while simulation parameters are summarized in Appendix A. We can see that the two models are in good agreement with the original data. The reason why these two models show such a similar behavior is that, despite their conceptually different architectures, all the necessary components which determine the interactions between the perceptual parts of the stimuli are similar. Indeed, all the processing stages are treated similarly and the different connectivity only provides a shortcut in the information pathways.

More details concerning the dynamics of the second network are illustrated in Fig. 7. Only the spatial cue task condition is shown, as it best represents the model's interesting characteristics. First, it can be seen in the cue integration layer that the selection process takes longer in the incongruent condition than in the congruent condition, as the selection mechanism must inhibit the movement cues. As for the motor selection areas it can be seen that ideomotor compatibility has a definite influence on RTs. Indeed, in the ideomotor compatible and congruent condition, the slope of the motor selection activity profile is the sharpest, indicating that the ideomotor region is facilitating the selection process. Conversely, in the incongruent condition, the ideomotor region facilitates the response in an opposite fashion to the one given by the cue integration layer, and hence slows down the final decision process. This interference effect is the cause of the increase in the RT.

\subsection{SR-incompatible mapping}

Since the previously reported simulation results of the two models are barely distinguishable (see Fig. 6), it shows us that the Brass et al. experimental paradigm cannot clearly discriminate between these two architectures. Therefore, it is important to devise a method for determining which model best 


\section{Single Route Model}

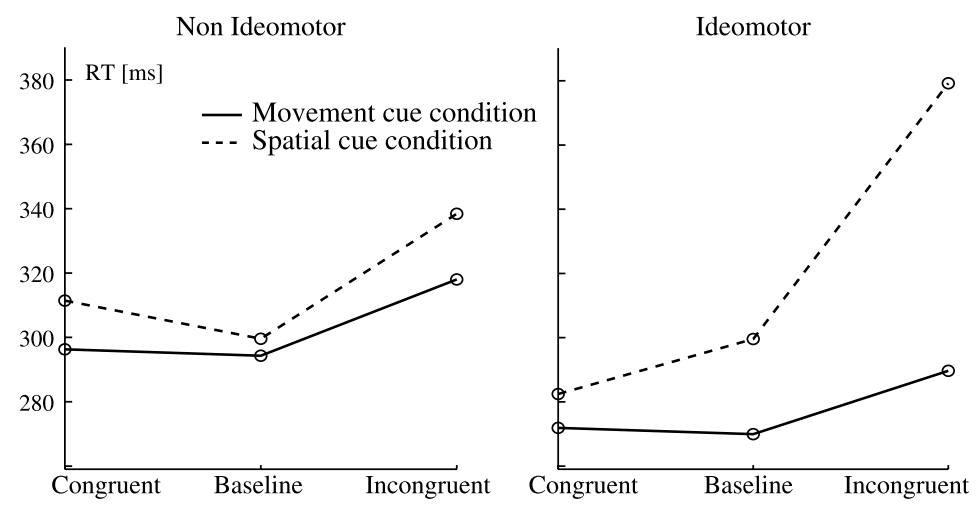

\section{Direct-Matching Model}
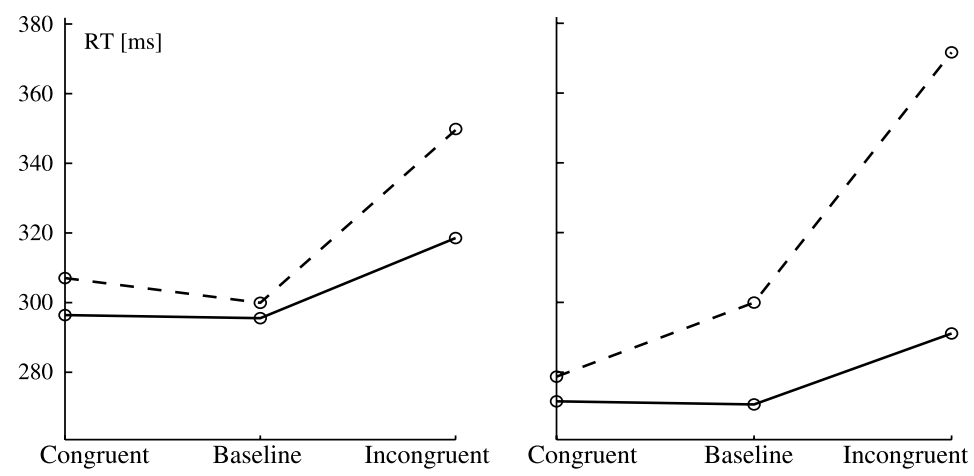

Fig. 6. Simulation results of our two model architectures under the same conditions as in the experiment by Brass et al. (2000). Both models are in good agreement with behavioral data (see Fig. 1).

\section{Spatial cue task}

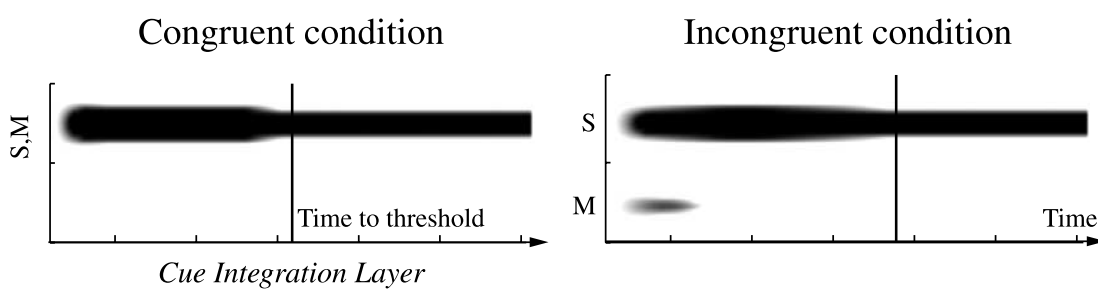

Ideomotor compatible
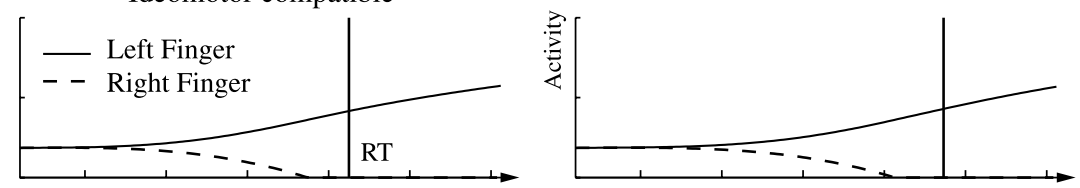

Motor Selection Layer

Ideomotor incompatible
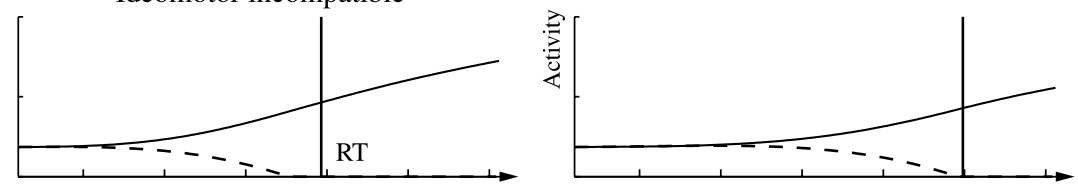

Motor Selection Layer

Fig. 7. Dynamics of the direct-matching model. The activity profiles of the cue integration population are shown on top. Dark areas correspond to regions of neural activity and the vertical lines denote the time at which the population activity reached a certain threshold. Labels S and $\mathrm{M}$ on the $y$-axis indicate the spatial location of spatial and movement cues, respectively. At the bottom, the time profile of the neuron with maximal activity of each motor selection area is shown. The vertical bar indicates the time at which the execution threshold $\delta$ was reached. 
(a)
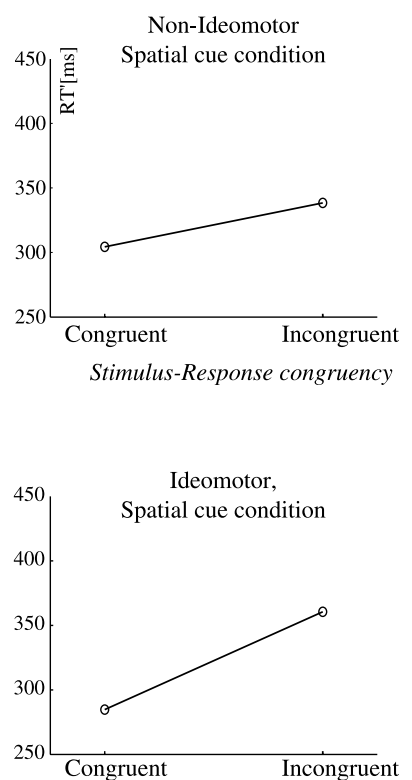

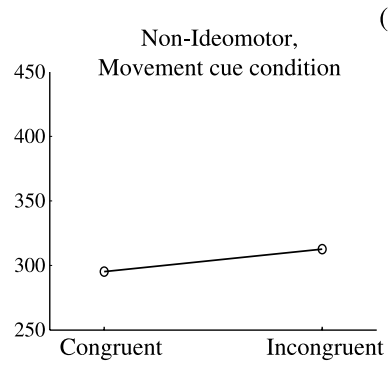

(b)
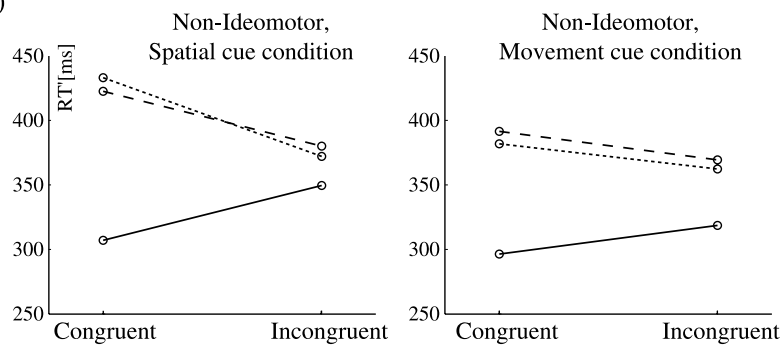

Compatible mapping

- - - Incompatible mapping (Single-route model)

........ Incompatible mapping (Direct-matching model)
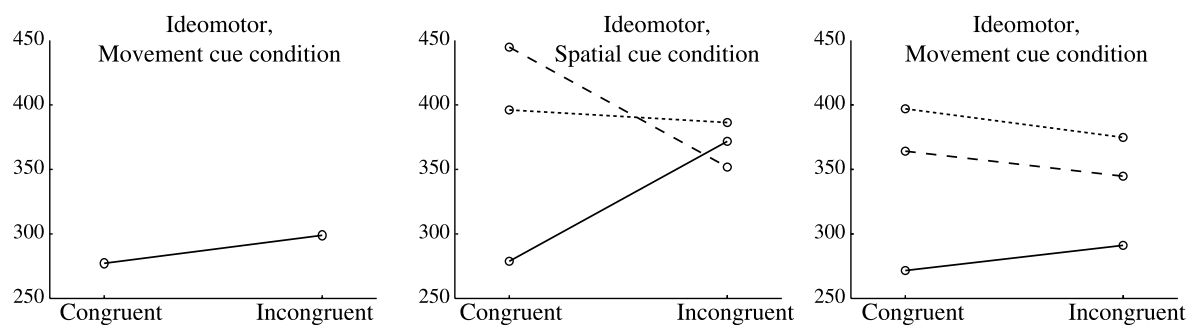

Fig. 8. (a) Results of Brass et al. (2000) redrawn, in order to account for the congruency of the irrelevant stimulus location with the response, as in a typical Hedge and Marsh experiment (Hedge \& Marsh, 1975). The baseline conditions were omitted to conform to that notation. The labels on the $x$-axis correspond to compatible and incompatible relationships of the irrelevant stimulus location with the response. (b) Predictive results of our models while confronted to an SR incompatible mapping task.

reflects the information pathway in the brain. Indeed, although both models produced similar results, the two model architectures are conceptually different.

To achieve this, we once again took inspiration from the large amount of literature on stimulus-response compatibility and decided to modify our models with an incompatible stimulus-response mapping. Such a mapping would be achieved by instructing our models to respond to a left cue with a motion of the right finger (middle finger), and conversely to respond to a right cue with a motion of the left finger (index finger). Such a set of instructions resembles a Simon-like task (Simon et al., 1981). Switching task instructions from a compatible to an incompatible stimulusresponse mapping results in a reversal of the classic Simon effect, ${ }^{5}$ as first reported by Hedge and Marsh (1975). Several explanations of this phenomenon have been proposed (Hasbroucq \& Guiard, 1991; Proctor \& Pick, 2003; Simon \& Berbaum, 1990). The most relevant to our argument is the stimulus-stimulus congruency hypothesis. The latter stresses that the facilitatory and interference effects are mainly caused by integration of spatial cues occurring in an intermediate processing level, rather than in late motor preparation stages. This hypothesis suggests that the reversal effect might be produced by a higher cognitive mechanism, involved in the incompatibility inversion process and that it should occur during the stimulus-response mapping process. This principle was implemented as suggested in Sections 2.1.2 and 2.3.4, by

\footnotetext{
${ }^{5}$ The Simon effect relates to the observation that even if the stimulus location is an irrelevant dimension, a spatial congruency between that irrelevant stimulus dimension and the response significantly facilitates the initiation of the response.
}

switching the wiring of the stimulus-response association module and by applying an additional processing time.

In Fig. 8a, we have redrawn the results of Brass et al. (2000), so as to account for the compatibility of the irrelevant stimulus location with the response, as in a typical Hedge and Marsh experiment (Hedge \& Marsh, 1975). In their experiment, Brass et al. did not explicitly distinguish stimulus-stimulus from stimulus-response congruency and we have to assume that these did not show any discordance.

Fig. $8 \mathrm{~b}$ shows the predictions of the two models. We can see that these two models exhibit different behaviors. The first model reproduces the classic reversal effect, i.e. the relative reaction times between the two conditions is reversed in contrast to the Brass et al. data. In other words, the RTs are faster when the irrelevant stimulus is located at the opposite side as that of the motor response. As for the second model, we observe qualitative differences. In the ideomotor and spatial cue condition, the reversal effect is reduced. This observation was expected as the direct route between action observation and action execution permanently activates the motor preparation centers in an ideomotor compatible way. Indeed, this effect can be seen in Fig. 9, where we compare the neural dynamics of the second model under SR compatible and incompatible mapping conditions. Results show that even in the incompatible mapping task an ideomotor compatible movement observation still strongly favors the corresponding movement execution, as the finger movement stimulus was unaffected by the task inversion process.

Moreover, in the ideomotor and movement cue condition, the overall RT is increased. A close look at Fig. 9 provides the explanation. First, the movement observation is used to determine the correct response, which is always on the 


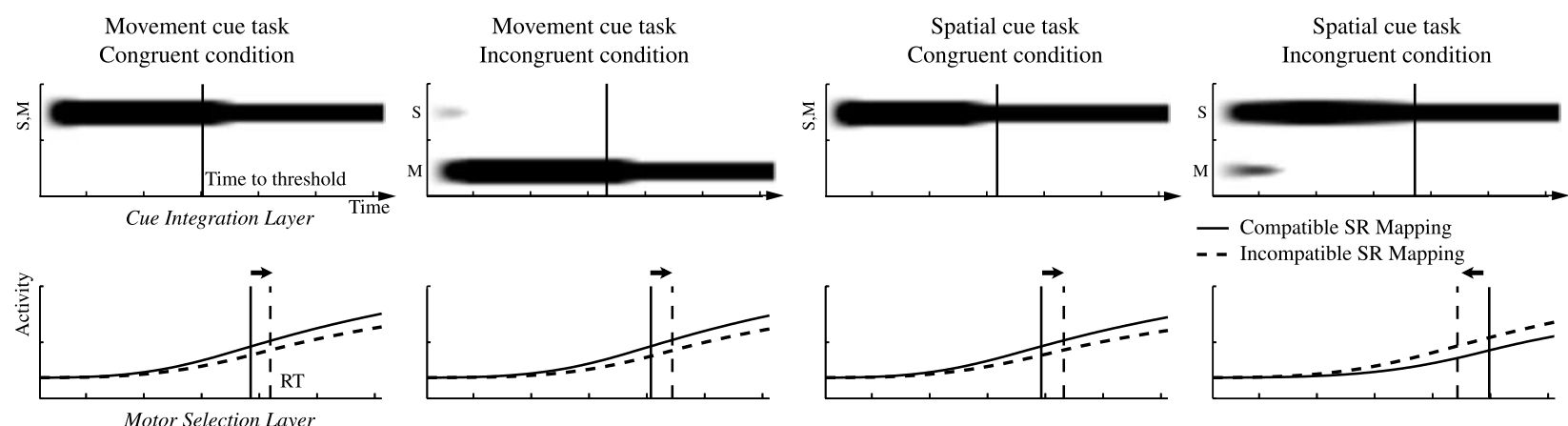

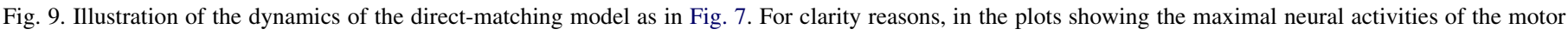

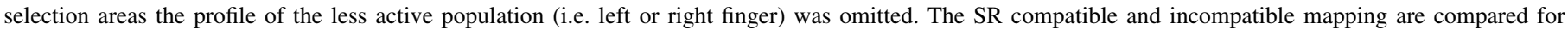

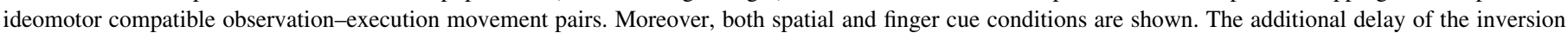

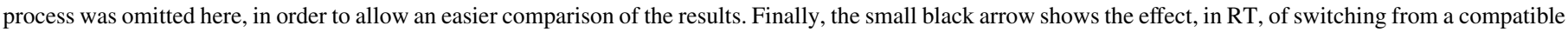
to an incompatible mapping task. For comments on the figure, see text.

opposite side. However, at the same time, the ideomotor system enhances the spatially matching finger movement, and as these two parallel processes always favor opposite responses, an interference effect is constantly present and results in an overall RT increase.

The reverse phenomena, although less significant, can be observed during the non-ideomotor conditions, see Fig. 8. Indeed, in the spatial cue condition, the Hedge and Marsh reversal effect increases slightly, whereas in the movement cue condition the overall RT decreases slightly. This can be explained by the fact that, in our models, when the observed/planned movement-pair is ideomotor incompatible, the network tends to favor, but to a lesser extent, the execution of the finger opposite the observed one. This opposite facilitatory effect therefore reverses the interference observed in the ideomotor condition.

\subsection{Metric of spatial representation}

Since the Dynamic Field Theory (DFT) was applied as a modeling framework, we present here our models predictions concerning a variation along the metric of spatial cue representation. The representation of spatial and motion cues used in the perception layer is considered continuous, rather than discrete. Therefore, as the DFT usually predicts modification in reaction times when stimuli are displaced according to their representation metric, we applied this principle in a new experimental paradigm.

The new paradigm consists of the same paradigm as employed by Brass et al. with the difference that the spatial cue, i.e. the cross, is no longer placed on a specific fingernail, but in a variable position between that fingernail and the midline of both fingers (see Fig. 10a). Simulation results showing the mean RT and the RT's difference between congruent and incongruent conditions as a function of the relative location of the spatial cue are presented in Fig. 10b. The horizontal axis represents the normalized spatial cue location so that a value of 0 corresponds to the fingernail position and 1 to the midline.

First, it can be seen that the mean RT of the two conditions behave in an opposite way. As the spatial cue moves toward the midline, its neural representation moves away from the finger location it corresponds to, and thus its interfering and triggering effect is reduced. This results in respective increase and decrease of the mean RT of the spatial and movement cues. If we consider now the variation of the RT difference between congruent and incongruent trials, the difference is reduced as the position of the spatial cue moves toward the center, confirming our previous claim. This can be explained in a similar fashion as when considering the mean RT above.

\section{Discussion}

In this paper, we presented two biologically inspired computational models capable of reproducing the experimental results obtained by Brass et al. (2000). These models are in line with other computational models addressing imitation mechanisms in both humans and monkeys (Arbib et al., 2000; Demiris \& Hayes, 2002; Wolpert et al., 2003), in that they all assume a shared representation between movement observation and action execution, which is mediated by competitive interactions. However, our models are also largely inspired by human behavioral phenomena reported in experimental psychology literature such as Hick's law, the Simon effect and the Hedge and Marsh reversal effect (Hedge \& Marsh, 1975; Hick, 1952; Simon et al., 1981).

More importantly, while the two models' architectures differed in their information pathway, they both successfully reproduced the ideomotor compatibility principle, based on a comparison of observed action with internally planned actions. The models complied with the definition of ideomotor compatibility, stating that if a perceptual event is similar to the response image that is used to control a response, then the perceived event should activate the response image and, hence, influence the initiation of the response (Brass et al., 2000; Greenwald, 1970; Wohlschläger et al., 2003).

In order to discriminate between these models, we proposed a novel stimulus-response experiment and presented our models' predictions. The experimental protocol of the experiment is similar to that conducted by Brass et al. (2000), except that the subjects should be asked to respond to any of the spatial cues with an incompatible response, i.e. they 
(a)

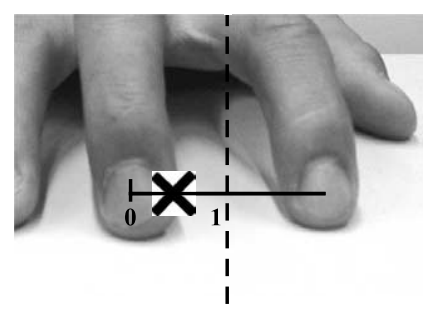

(b)
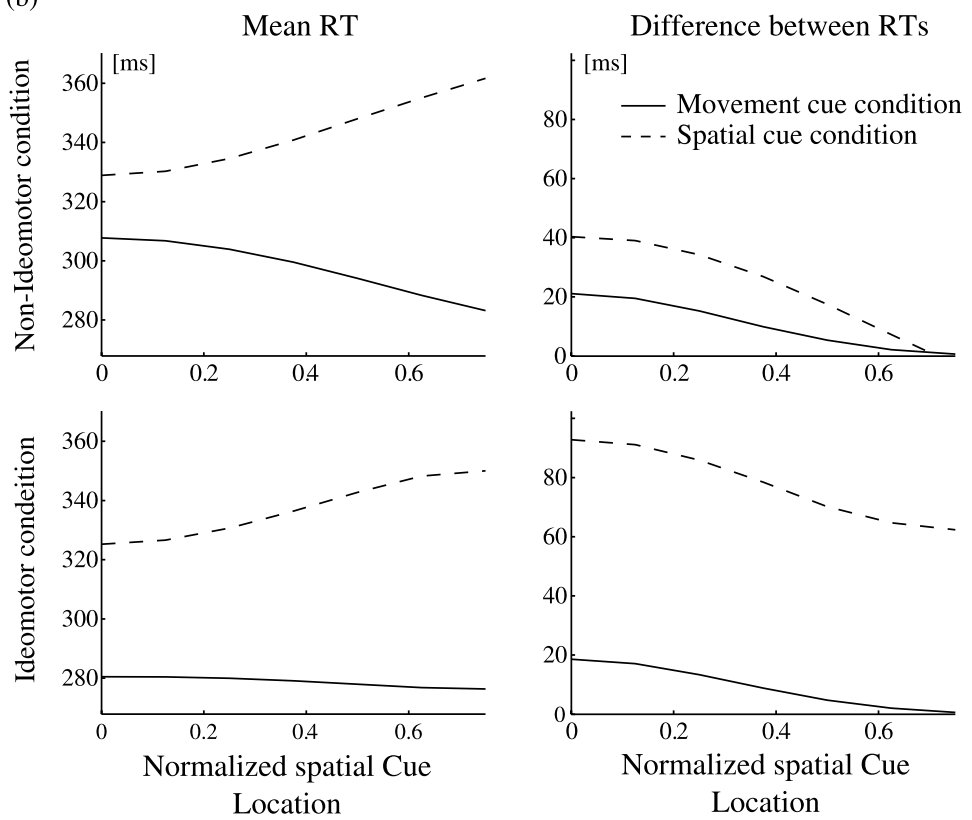

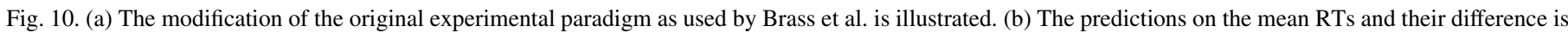
shown, while varying the location of the spatial cue.

should respond to a left (right) cue with a right (left) finger movement. If that experiment was to be conducted and a strong Hedge and Marsh reversal effect was to be measured, then this would refute our direct-matching model and let the single-route model appear to be more plausible.

The direct-matching model relies on the hypothesis that a direct route between movement observation and movement execution exists (Decety \& Sommerville, 2003; Rizzolatti et al., 2001). In other words, the model hypothesizes that the spatial relationships between relevant and irrelevant stimuli is processed first at an intermediate level, in brain areas primarily concerned with task constraints. While the comparison between planned and observed motions is conducted separately but simultaneously through an ideomotor pathway, before both pathways get merged.

We note that, if one would not observe the opposite of the Hedge and Marsh reversal effect, this may not necessarily refute the plausibility of the direct-matching model. Since the direct and automatic imitative response in a simple stimulus-response (SR), incompatible mapping tasks can be easily overridden after only a brief training (Heyes et al., 2005). Therefore, it will be essential that the experiment be performed with subjects who remain totally unfamiliar with the task. Subsequently, it would be interesting to see if such SR incompatible training results in an effective suppression of the ideomotor effect.
The results of our simulations showed that the observation of a non-ideomotor compatible finger movement results in a slight facilitation of the initiation of the opposite finger. This anti-facilitatory effect seems questionable, as such a phenomenon has never been observed in the corresponding literature. This may be a weakness of our model. The main reason for such effects to occur in our model is that, in the motor selection area, the process of ideomotor facilitation and interference acts within the same metric as that of the decisional layer, i.e. by considering the global amplitude of the population activity. Then, as the competition among the motor plans is performed in that metric, the decrease in amplitude caused by an ideomotor incompatible observed movement favors the execution of the opposite finger's motor plan. This problematic issue raises the question of how the brain represents such multimodal information.

Indeed, the last and unexplored hypothesis is effectively that of the role taken by the metric of stimulus representation. By assuming continuous representations, we do not claim here that such unique and distinct representations exist as it is in the brain, but this simplification is mainly used for convenience. Indeed, it is not within the scope of this paper to address the question of how distributed representations can emerge from sensory receptors and cortical interactions. This topic is left for future work. Similar questions arise when considering the 
metric of movement representation. In our modeling approach, we assumed that the movements performed by others are represented and encoded within the same frame of reference as self-generated movements, in accordance with the directmatching hypothesis and the firing patterns of mirror neurons. This computational issue was already addressed in Pouget et al. (2003) and Sauser and Billard (2005). We also assumed that opposite finger movements, such as tapping and lifting, are encoded by means of directional information within the same neural layer. This suggests that the spatial relationships at stake in our experiments are encoded in a limb-centered frame of reference. This later hypothesis remains yet to be verified.

Arguments in favor of this hypothesis are, for instance, the fact that there exist direct pathways from the primary motor cortex and the premotor cortex to the control of finger motion, that the premotor cortex is involved in imitation of finger movements (Iacoboni et al., 1999), and that movements in the primary and premotor cortex are sometimes encoded by means of directional information (Cisek \& Kalaska, 2005; Schwartz et al., 1988).

\section{Conclusion}

In this paper, we have presented two biologically plausible neural architectures addressing the effect of ideomotor compatibility between observed and executed movements as reported in the behavioral experiment by Brass et al. (2000). These networks were developed along a multidisciplinary approach linking neuroscience with experimental psychology, which tries to bridge the gab between human behavioral data and single cell recordings in monkeys. Then, despite the different information pathways used by these two models, they were both fairly capable of reproducing the experimental results mentioned above.

Furthermore, the models have been subjected to two novel experimental conditions in order to (1) investigate the metric of stimulus representation in such a task and (2) to determine which of the two models might be the most plausible.

\section{Acknowledgements}

This work was supported by the Swiss National Science Foundation, through grant no. 620-066127 of the SNF Professorships program.

\section{Appendix A. Simulation parameters}

The parameters used in the simulations of our two models as illustrated in Fig. 6, are summarized in Table A1.

Simulated RTs $X$ were fitted to the original data $Y$ using a first order least squares error regression method. The estimated RTs $\hat{X}$ are given by

$\hat{X}=c_{1} X+c_{2}$

where the constants $c_{1}$ and $c_{2}$ were determined so that the error
Table A1

Simulation parameters

\begin{tabular}{|c|c|c|c|}
\hline & Parameter & Single-route & Direct-matching \\
\hline \multicolumn{4}{|l|}{ Perceptual layer } \\
\hline \multicolumn{4}{|l|}{ MST region } \\
\hline \multirow{3}{*}{ Spatial cue } & $\alpha$ & 1.5 & 1.53 \\
\hline & $\beta$ & 0.88 & 0.9 \\
\hline & $\phi$ & $\pm \pi / 2$ & $\pm \pi / 2$ \\
\hline \multirow[t]{3}{*}{ Motion cue } & $\alpha$ & 1.5 & 1.53 \\
\hline & $\beta$ & 0.97 & 1.0 \\
\hline & $\phi$ & $\pm \pi / 2$ & $\pm \pi / 2$ \\
\hline $\begin{array}{l}\text { Top-down } \\
\text { modulation }\end{array}$ & $h^{T}$ & 0.26 & 0.2 \\
\hline \multirow{2}{*}{\multicolumn{4}{|c|}{ inhibition }} \\
\hline & & & \\
\hline \multicolumn{4}{|l|}{ STS region } \\
\hline Movement & $\alpha$ & 0.0 & 0.0 \\
\hline \multirow[t]{2}{*}{ observation } & $\beta$ & 0.79 & 0.5 \\
\hline & $\phi$ & $\pm \pi / 2$ & $\pm \pi / 2$ \\
\hline \multicolumn{4}{|l|}{ Decision layer } \\
\hline \multicolumn{4}{|l|}{ PPC region } \\
\hline \multirow[t]{5}{*}{ Cue integration } & $\alpha$ & 1.02 & 2.0 \\
\hline & $\gamma_{\text {SpaCue } \rightarrow \text { CueInt }}$ & 1.16 & 1.65 \\
\hline & $\gamma_{\text {MvtCue } \rightarrow \text { CueInt }}$ & 1.16 & 1.65 \\
\hline & $\gamma_{\text {IdeInt }(\mathrm{L} / \mathrm{R})} \rightarrow$ & 8.95 & 1.65 \\
\hline & CueInt & & \\
\hline \multicolumn{4}{|c|}{ Motor preparation layer } \\
\hline \multicolumn{4}{|l|}{ PMd region } \\
\hline \multirow[t]{3}{*}{ Motor plan (L/R) } & $\alpha$ & 0.0 & 0.0 \\
\hline & $\beta$ & 1.0 & 1.0 \\
\hline & $\phi$ & $\pm \pi / 2$ & $\pm \pi / 2$ \\
\hline \multicolumn{4}{|l|}{ PMv (F5) region } \\
\hline \multirow{5}{*}{$\begin{array}{l}\text { Ideomotor } \\
\text { integration }(\mathrm{L} / \mathrm{R})\end{array}$} & $\alpha$ & 1.98 & 1.81 \\
\hline & $\gamma_{\text {MotPlan(L/R })} \rightarrow$ & 1.08 & 1.19 \\
\hline & $\operatorname{IdeInt}(\mathrm{L} / \mathrm{R})$ & & \\
\hline & $\gamma_{\operatorname{MotObs}(\mathrm{L} / \mathrm{R})} \rightarrow$ & 1.08 & 1.19 \\
\hline & $\operatorname{IdeInt(L/R)}$ & & \\
\hline \multicolumn{4}{|l|}{$\mathrm{PMv}(\mathrm{F} 4)$ region } \\
\hline \multirow{6}{*}{$\begin{array}{l}\text { Motor selection } \\
(\mathrm{L} / \mathrm{R})\end{array}$} & $\alpha$ & 0.15 & 0.09 \\
\hline & $\gamma_{\text {CueInt(L/R) }} \rightarrow$ & 3.91 & 4.02 \\
\hline & $\operatorname{Motsel}(\mathrm{L} / \mathrm{R})$ & & \\
\hline & $\gamma_{\text {MotPlan(L/R })} \rightarrow$ & 1.58 & 4.02 \\
\hline & $\operatorname{Motsel(L/R)}$ & & \\
\hline & $\gamma_{\text {IdeInt(L/R }) \rightarrow}$ & & 1.58 \\
\hline $\begin{array}{l}\text { Reciprocal } \\
\text { inhibition }\end{array}$ & $\begin{array}{l}\text { Motsel(L/R) } \\
W_{\text {Motor }}^{M}\end{array}$ & 7.71 & 7.34 \\
\hline $\begin{array}{l}\text { Execution } \\
\text { threshold }\end{array}$ & $\delta$ & 0.08 & 0.08 \\
\hline \multicolumn{4}{|l|}{ Other constants } \\
\hline \multirow[t]{3}{*}{ Variance profile } & $\sigma$ & 0.3 & 0.3 \\
\hline & $\sigma_{\text {CueInt(L/R }) \rightarrow}$ & 0.5 & 0.5 \\
\hline & $\operatorname{MotSel(L/R)~}$ & & \\
\hline Time constant & $\tau$ & 0.1 & 0.1 \\
\hline $\begin{array}{l}\text { Additional time } \\
\text { delay }(\mathrm{ms})\end{array}$ & $\Delta$ & & \\
\hline $\begin{array}{l}\text { Compatible } \\
\text { mapping } \\
\text { condition }\end{array}$ & & 0.0 & 0.0 \\
\hline $\begin{array}{l}\text { Incompatible } \\
\text { mapping } \\
\text { condition }\end{array}$ & & 73.0 & 73.0 \\
\hline Regression & $c_{1}$ & 3292.19 & -263.32 \\
\hline constants & $c_{2}$ & 1770.54 & -69.21 \\
\hline
\end{tabular}


$E=\|Y-\hat{X}\|^{2}$

is minimized.

Concerning the choice of the simulations parameters, they were initially tuned according to our work hypotheses as presented in Section 2.1. Then, they were fine-tuned using a gradient descent method in order to minimize the error (Eq. (A2)) between the behavioral data and the simulation results.

\section{References}

Amari, S. I. (1977). Dynamics of pattern formation in lateral-inhibition type neural fields. Biological Cybernetics, 27, 77-87.

Andersen, R. A., Snyder, L. H., Bradley, D. C., \& Xing, J. (1997). Multimodal representation of space in the posterior parietal cortex and its use in planning movements. Annual Review Neuroscience, 20, 303-330.

Arbib, M., \& Bota, M. (2003). Language evolution: Neural homologies and neuroinformatics. Neural Networks, 16, 1237-1260.

Arbib, M., Billard, A., Iacoboni, M., \& Oztop, E. (2000). Mirror neurons, imitation and (synthetic) brain imaging. Neural Networks, 13, 953-973.

Ashbridge, E., Perrett, D. I., Oram, M. W., \& Jellema, T. (2000). Effect of image orientation and size on object recognition: Responses of single units in the macaque monkey temporal cortex. Cognitive Neuropsychology, 17, $13-34$.

Billard, A. (2002). Imitation. In M. A. Arbib (Ed.), The handbook of brain theory and neural networks (pp. 566-569). Cambridge, MA: MIT Press.

Billard, A., \& Mataric, M. (2001). Learning human arm movements by imitation: Evaluation of a biologically-inspired connectionist architecture. Robotics and Autonomous Systems, 941, 1-16.

Brass, M., Bekkering, H., Wohlschläger, A., \& Prinz, W. (2000). Compatibility between observed and executed finger movements: Comparing symbolic, spatial and imitative cues. Brain and Cognition, 44, 124-143.

Brass, M., Bekkering, H., \& Prinz, W. (2001). Movement observation affects movement execution in a simple response task. Acta Psychologica, 106, $3-22$.

Cisek, P., \& Kalaska, J. F. (2005). Neural correlates of reaching decision in dorsal premotor cortex: Specification of multiple direction choices and final selection of action. Neuron, 45, 801-814.

Decety, J., \& Sommerville, J. A. (2003). Shared representations between self and others: A social cognitive neuroscience view. Trends in Cognitive Sciences, 7, 527-533.

Demiris, Y., \& Hayes, G. (2002). Imitation as a dual process featuring predictive and learning components: A biologically plausible computational model. In Imitation in animals and artefacts (pp. 327-362) MIT Press.

Erlhagen, W., \& Schöner, G (2002). Dynamics field theory of movement preparation. Psychological Review, 109(3), 545-572.

Franconeri, S. L., \& Simons, D. L. (2005). The dynamic events that capture visual attention: A reply to Abrams and Christ. Perception and Psychophysics, 67, 962-966.

Greenwald, A. G. (1970). Sensory feedback mechanisms in performance control: With special reference to the ideo-motor mechanism. Psychological Review, 77(2), 73-99.

Hasbroucq, T., \& Guiard, T. (1991). Stimulus-response compatibility and the Simon effect: Toward a conceptual clarification. Journal of Experimental Psychology: Human Perception and Performance, 17(1), 266-267.

Hedge, A., \& Marsh, N. W. A. (1975). The effect of irrelevant spatial correspondences on two-choice response-time. Acta Psychologica, 39, 427-439.

Heyes, C., Bird, G., Johnson, H., \& Haggard, P. (2005). Experience modulates automatic imitation. Cognitive Brain Research, 22, 233-240.

Hick, W. E. (1952). On rate of gain of information. The Quarterly Journal of Experimental Psychology, 4, 11-26.
Iacoboni, M., Woods, R. P., Brass, M., Bekkering, H., Mazziotta, J. C., \& Rizzolatti, G. (1999). Cortical mechanisms of human imitation. Science, 286, 2526-2528.

Itti, L., \& Koch, C. (2001). Computational modeling of visual attention. Nature Reviews Neuoscience, 2, 193-203.

Jellema, T., Maassen, G., \& Perrett, D. I. (2004). Single cell integration and aminate form, motion and location in the superior temporal cortex of the macaque monkey. Cerebral Cortex, 14, 781-790.

Kilner, J. M., Paulignan, Y., \& Blakemore, S. J. (2003). An interference effect of observed biological movement on action. Current Biology, 13, 522-525.

Kopecz, K., \& Schöner, G. (1995). Saccadic motor planning by integrating visual information and pre-information on neural dynamic fields. Biological Cybernetics, 73, 49-60.

Kornblum, S. (1994). The way irrelevant dimensions are processed depends on what they overlap with: The case of Stroop- and Simon-like stimuli. Psychological Research, 56, 130-135.

Meltzoff, A., \& Moore, M. (1997). Explaining facial imitation: A theoretical model. Early Development and Parenting, 6, 179-192.

Oztop, E., Kawato, M., \& Arbib, M. (2006). Mirror neurons and imitation: A Computationally Guided Review. Neural Networks, 19(3), doi:10.1016./j.nuenet.2006.02.002

Perrett, D. I., Harries, M., Mistlin, A. J., \& Chitty, A. J. (1989). Three stages in the classification of body movements by visual neurons. In H. B. Barlow, C. Blakemore, \& M. Weston-Smith (Eds.), Images and understanding (pp. 94-107). Cambridge: Cambridge University Press.

Pouget, A., Dayan, P., \& Zemel, R. S. (2003). Inference and computation with population codes. Annual Reviews Neuroscience, 26, 381-410.

Proctor, R. W., \& Pick, D. F. (2003). Display-control arrangement correspondence and logical recoding in the Hedge and Marsh reversal of the Simon effect. Acta Psychologica, 112, 259-278.

Rizzolatti, G., Fogassi, L., \& Gallese, V. (2001). Neurophysiological mechanisms underlying the understanding of actions. Nature Reviews Neuroscience, 2, 661-670.

Salinas, E., \& Abbott, L. F. (1996). A model of multiplicative neural response in parietal cortex. Proceedings of the National Academy of Sciences USA, 93, 11956-11961.

Sauser, E. L., \& Billard, A. G. (2005). Three dimensional frames of references transformations using recurrent populations of neurons. Neurocomputing, $64,5-24$.

Schadlen, M. N., \& Newsome, W. T. (2001). Neural basis of perceptual decision in the parietal cortex (area LIP) of the rhesus monkey. Journal of Neurophysiology, 86, 1916-1936.

Schwartz, A. B., Kettner, R. E., \& Georgopoulos, A. P. (1988). Primate motor cortex and free arm movements to visual targets in three-dimensional space: I. relations between single cell discharge and direction of movement. Journal of Neuroscience, 8(8), 2913-2927.

Simon, J. R., \& Berbaum, K. (1990). Effect of conflicting cues on information processing: The Stroop effect vs. the Simon effect. Acta Psycologica, 73, 159-170.

Simon, J. R., Sly, P. E., \& Vilapakkam, S. (1981). Effect of compatibility of S$\mathrm{R}$ mapping on reactions toward the stimulus source. Acta Psychologica, 47, 63-81.

Wilimzig, C., \& Schöner, G. (2005). The emergence of stimulus-response associations from neural activation fields: Dynamic field theory. In Proceedings of the XXVIII annual conference of the cognitive science society (CogSci 2005), Stresa, Italy, (pp. 2359-2364).

Wohlschläger, A., Gattis, M., \& Bekkering, H. (2003). Action generation and action perception in imitation: An instance of the ideomotor principle. Philosophical Transactions of the Royal Society of London, 358, 501-515.

Wolpert, D. M., Doya, K., \& Kawato, M. (2003). A unifying computational framework for motor control and social interaction. Philosophical Transactions of the Royal Society of London, 358, 593-602.

Zhang, H. H., Zhang, J., \& Kornblum, S. (1999). A parallel distributed processing model of stimulus-stimulus and stimulus-response compatibility. Cognitive Psychology, 38, 386-432.

Zhang, K. (1996). Representation of the spatial orientation by the intrinsic dynamics of the head-direction cell ensemble: A theory. Journal of Neuroscience, 16(6), 2112-2126. 